\title{
Attenuated cortisol reactivity to psychosocial stress is associated with greater visual dependency in postural control
}

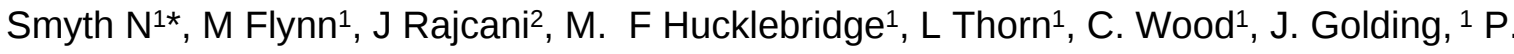 \\ Evans ${ }^{1}$ and $A$ Clow $^{1}$. \\ 1Psychophysiology and Stress Research Group, Psychology, School of Social Sciences, \\ University of Westminster, 115 New Cavendish Street, London W1W 6UW, UK. \\ ${ }^{2}$ Department of Psychology, Faculty of Arts, Comenius University, Gondova 2, 81499 \\ Bratislava, Slovakia.
}

*Corresponding author: N.Smyth@westminster.ac.uk. Psychophysiology and Stress Research Group, School of Social Sciences, University of Westminster, 115 New Cavendish Street, London W1 6UW, UK.

Keywords: Vestibular function; postural control; TSST-G; cortisol stress reactivity; visual dependency 
- HPA-axis activity related to vestibular function in healthy participants

- Attenuated cortisol reactivity related to visual dependency in postural control

- Non-clinical differences in vestibular function is related to HPA axis dysfunction 


\begin{abstract}
Despite known anatomical links between the hypothalamic-pituitary-adrenal (HPA) axis and the vestibular system, there are no studies on the relationship between postural control and HPA axis function. Visual dependence in postural control, often measured by increased postural sway on exposure to visual motion, is an indication of altered visual-vestibular integration with greater weighting towards visual cues for balance. Visual dependence is more common in older age and a range of vestibular and non-vestibular health conditions. The relationship between visual dependence in postural control was investigated in relation to cortisol reactivity to psychosocial stress (using the Trier Social Stress Test for groups: TSST-G), as an index of HPA axis function, in healthy young females. In those who exhibited a cortisol response ( $>2 \mathrm{nmol} / \mathrm{l})$, a negative relationship between stress-induced cortisol reactivity and visual dependence in postural control was observed, since those with the largest cortisol response showed less visual motion induced postural sway (measured by force platform). This finding in healthy females indicates that subtle non-clinical differences in vestibular function are associated with dysregulated HPA axis activity as indicated by lower cortisol reactivity to psychosocial stress. It adds to the growing body of evidence linking blunted cortisol reactivity to stress to poor homeostatic regulation and potential negative health and behavioural outcomes.
\end{abstract}




\section{Introduction}

Postural control is dependent upon the integration of information from the proprioceptive, vestibular and visual sensory systems. There are individual differences in the degree of reliance upon each of these inputs. Visual dependence results from additional reliance on visual signals when vestibular signals are absent or disordered. Visual dependence can be associated with postural instability during exposure to complex moving visual stimuli (Redfern et al., 2001). High visual dependence may not necessarily lead to postural instability as people can also use haptic cues to aid stability. It is however more common with older age and a range of vestibular and non-vestibular health conditions such as vestibular neuritis, anxiety, type-2 diabetes, schizophrenia and Parkinson's disease (Cousins et al., 2014; Jeon et al., 2007; Razzak and Hussein, 2016). There are documented links between vestibular dysfunction and anxiety disorders (Furman and Jacob, 2001) with these patient groups showing increased visual dependence (Redfern et al., 2001). In addition, vestibular dysfunction, is linked to a variety of cognitive impairments such as executive function, and memory (Bigelow and Agrawal, 2015). It is noteworthy that the range of conditions associated with increased visual dependency shows some parallels with hypothalamic pituitary adrenal (HPA) axis dysfunction of (e.g. Bradley and Dinan, 2010; Chan et al., 2003; Faravelli et al., 2012; Hartmann et al., 1997). Indeed, the vestibular system and HPA axis have bi-directional anatomical links (Markia et al., 2008; Saman et al., 2012). Stress can influence vestibular function and vestibular dysfunction induces activation of the HPA axis (Saman et al., 2012; Yamanaka et al., 1995). Moreover, patients with persistent vestibular dysfunction have high cortisol levels, although the direction of causality is not clear (Martin et al., 2016; Saman et al., 2012). Despite these known links, there are no reported studies investigating the relationship between cortisol stress reactivity, an index of HPA axis function, and visual dependence in regulation of postural control.

Postural control can be assessed in the laboratory by measurement of sway path length whilst standing on a force plate. Visual field signals project via the cerebellum to secondary vestibular neurones in the brainstem, summating with vestibular afference, and thence control of posture via vestibulo-spinal pathways. Visual dependence can be determined by sway path length during exposure to a moving visual environment. A stimulus commonly used to test this phenomenon is the rod and disk, consisting of a projected collage of small white dots on a black background, presented either stationary or rotated (Cousins et al., 2017). This provides an index of sensitivity to a moving visual stimulus in terms of worse postural sway but it is not a measure of instability per se. 
HPA axis function can be characterised by measurement of cortisol reactivity to an acute stress challenge or aspects of the underlying diurnal pattern of cortisol secretory activity. Stress reactivity can be assessed within the laboratory setting using a range of physical (e.g. the cold pressor test) and/or psychological paradigms, such as the mirror drawing and STROOP tests (for an overview see Zänkert et al., 2018). Of these the Trier Social Stress Test (TSST) (Kirschbaum et al., 1993) has been shown to most reliably induce activation of the HPA axis (Dickerson and Kemeny, 2004; Goodman et al., 2017; Kirschbaum et al., 1993; Skoluda et al., 2015). The individual TSST (Kirschbaum et al., 1993) has been adapted for use with groups (TSST-G: Kirschbaum et al., 1993; von Dawans et al., 2011) and has been used successfully in a range of studies in healthy populations. Large inter-individual variability in trait stress reactivity is related to a range of demographic (e.g. age and sex) and psychosocial factors in healthy participants. Typically, larger cortisol responses are associated with disadvantage such as low self-esteem, low birthweight, and anxious attachment style (see Kudielka et al., 2009; Smyth et al., 2015). However, more recently blunted (defined as a smaller increase from base to peak) stress reactivity is just as indicative of poor homeostatic regulation as exaggerated responding. It has been implicated in a range of different adverse behavioural and health outcomes in clinical and healthy populations. For example, blunted stress reactivity has been associated with anxiety disorders, low socioeconomic status, poorer cognitive performance and smaller hippocampal volume, as well as in female veterans regardless of their post-traumatic stress disorder status (Almela et al., 2014; Carroll et al., 2017; Domes et al., 2002; Pierce and Pritchard, 2016; Raffington et al., 2018).

In this study, we examined, for the first time, the relationship between visual dependence in postural control and cortisol reactivity to the TSST-G, as a marker of HPA axis function, in healthy females. Known links between vestibular and HPA axis function alongside evidence of impaired cognitive function in both visual dependence and HPA axis dysregulation provided the rationale for the investigation. We predicted lower stress-induced cortisol reactivity would be associated with greater visual dependence in postural control.

\section{Methods}

\subsection{Participants}

Females $(N=74)$ were recruited from the academic community on the basis that they were healthy, not taking steroid medication or suffering from diagnosed physical or psychiatric 
illness (ascertained by self-report). They received either a shopping voucher or course credits for their participation. An all-female sample was recruited to control for sex differences in cortisol stress reactivity (see Smyth et al., 2013). Data were missing for three participants: cortisol $(n=2)$ and postural sway $(n=1)$. Following removal of outliers $(>3$ standard deviations from the mean) analyses were conducted on 68 participants. Of those that reported their demographics or health status descriptive statistics are provided in Table 1. The University of Westminster Ethics Committee approved the protocol and all participants provided written informed consent.

\section{Insert Table 1 about here}

\subsection{Materials and Procedure}

Participants completed an online screening form and invited to attend a research session in randomly selected groups of up to 6 participants. The size of groups ranged between 3 and 6 participants, most groups included 5 participants (mode $=5$ ). During this session, they were introduced to the saliva-sampling method and asked to record the date of their last menstruation. Participants individually completed the postural sway testing followed by the group version of Trier Social Stress Test (TSST-G: von Dawans et al., 2011) detailed below.

All testing took place in the afternoon, when there is less change in salivary cortisol concentrations, after the morning cortisol peak that could interfere with stress responses (see. Smyth et al., 2013). The TSST-G included the group preparatory period (30 min), the group stress task (22 $\mathrm{min}$ ), and the group recovery and debriefing period (40 min). During the preparatory phase, participants were free to talk with each other, and were given $10 \mathrm{~min}$ to prepare notes for a $2 \mathrm{~min}$ free speech to be given to a committee. They were instructed to introduce themselves to the committee and convince them that they were the most suitable candidates for a given job. During the group stress task phase, all participants were taken into another room with an already seated committee comprising of one female and one male wearing white laboratory coats. There were two conspicuous video cameras. In a random order, participants were asked to give free speech followed by the mental arithmetic task, which was to serially subtract the number 17 from a given 4-digit number for 80 seconds. The committee members gave standard responses to participants who finished their speech before the 2 min duration or failed in the subtraction task. Participants returned to Room 1 for the recovery period and debrief. Figure 1 illustrates the experimental timeline. 
Saliva samples were collected under the supervision of a researcher and participants were asked to remain nil-by-mouth (except water) and not to exercise or smoke for 30 min prior to testing. Two baseline samples were collected, the first prior to the postural sway testing and the second immediately before the TSST-G task period. Samples were collected immediately after the speech task (S2, at $12 \mathrm{~min}$ ), after the mental arithmetic task (S3, at 22 $\mathrm{min}$ ), and every $10 \mathrm{~min}$ up to $60 \mathrm{~min}$ (S4, at $32 \mathrm{~min} \mathrm{S5}$, at $42 \mathrm{~min}, \mathrm{~S} 6$, at $52 \mathrm{~min}$, and S7, at $62 \mathrm{~min})$.

\subsection{Assessment of visual dependence in postural sway}

Black-out blinds and consistent artificial light was used to ensure consistency in presentation of the visual stimuli. Participants stood on a force platform in front of a display panel (height $180 \mathrm{~cm}$, width $240 \mathrm{~cm}$ size), positioned approximately $120 \mathrm{~cm}$ away. To restrict peripheral visual cues and to focus participants' gaze on the stimulus, participants wore a pair of lightweight googles, and were instructed to stand with feet together, and arms by their sides. For 60 secs participants performed the Rod-and-Disk task. This consists of a luminous white $6 \mathrm{~cm}$ rod, which can rotate in either direction about its midpoint in the central $11^{\circ}$ of the visual field. Surrounding this central point is a black background with a collage of 220 offwhite dots, each $8 \mathrm{~mm}\left(1.5^{\circ}\right.$ of visual field) in diameter, randomly distributed. This was performed under two conditions, visual static (dots stationary) or visual motion (dots rotating) (see Cousins et al., 2017). In each condition, postural sway path length was measured in $\mathrm{cm}$ on a stable force platform (Accusway AMTI Ltd). Total postural sway path length was divided by the duration of the trial to obtain average sway path length $(\mathrm{cm} / \mathrm{sec})$, with higher values indicative of greater postural sway. On an individual basis, participants completed measures of postural sway in in a separate room from the TSST-G. Testing took approximately $10 \mathrm{~min}$ per participant (see figure 1).

\section{Insert Figure 1 about here}

\subsection{Salivary cortisol assessment}

Samples were stored at $-20{ }^{\circ} \mathrm{C}$ in the laboratory until assayed. They were thawed and centrifuged for 10-15 min at 3,500 rpm. Cortisol concentrations were determined by enzyme linked immunosorbent assays (Salimetrics LLC, State College, PA) at the Psychophysiology and Stress Research Group's laboratory. Standards, controls and all samples were assayed in duplicate and intra and inter-assay variations were both below $10 \%$.

\subsection{Treatment of Data \& Statistical Analysis}


Cortisol values were positively skewed and were transformed to normalise. Data were omitted for two participants exhibiting exceptionally high cortisol reactivity ( $>3$ standard deviations from the mean). A repeated measures analysis of variance (ANOVA) was used to examine differences in cortisol concentrations, in all participants. As participants performed the TSST-G tasks at slightly different time-points, cortisol stress reactivity was calculated as the individual peak sample (from samples 3-8) minus individual baseline sample, derived from the first two baseline samples (Miller et al., 2018). Individuals who showed at least 2.0 $\mathrm{nmol} / \mathrm{L}$ increase in cortisol reactivity were defined as cortisol responders, and the remainder were categorized as non-responders. A mixed ANOVA was conducted to illustrate differences in cortisol concentrations between the cortisol responders and non-responders. Pairwise comparisons were conducted to examine differences in cortisol concentrations between the two groups. Postural sway data in both conditions (static, motion) were positively skewed, and therefore data was transformed. Data for one participant for exceptionally high postural sway (>3 standard deviations from the mean) was omitted. For illustration purposes, untransformed data are presented in the table and figures.

Regression analysis was conducted to examine the effect of postural sway on cortisol reactivity for both the visual static and motion conditions. We also modelled the interaction term between postural sway and cortisol responding status (responder vs non-responder) in order to assess whether the relationship between postural sway and cortisol reactivity might depend on whether or not participants exhibited a cortisol response to the TSST-G. Additional predictors (e.g. menstrual cycle, age, smoking status and BMI) were also included in the model to assess if effects remained.

\section{Results}

Raw values for cortisol concentration ranged from 0.85 to $13.48 \mathrm{nmol} / \mathrm{l}$. A repeated measures ANOVA revealed that, in the whole sample, a cortisol response was induced by the TSST-G $\left(F_{(3.1,207.6)}=8.828, p<.001\right)$. Within-subjects contrasts revealed a significant quadratic effect $\left(F_{(1,66)}=17.299, p<.001\right)$, such that on average cortisol increased from baseline and peaked $10 \mathrm{~min}$ following the TSST-G tasks (sample 4) and declined thereafter. Cortisol reactivity composite was calculated, as individual peak sample minus individual baseline sample. There was an average cortisol response $(M=2.0, S D=2.66 \mathrm{nmol} / \mathrm{l})$ following onset of the stressor, and 35\% ( $\mathrm{N}=24)$ were classified as a cortisol responder (i.e. $>2.0 \mathrm{nmol} / \mathrm{L}$ increase). Cortisol reactivity was obviously greater for the cortisol responders $(M=5.0, S D=2.3 \mathrm{nmol} / \mathrm{l})$ compared to the non-responders $(\mathrm{M}=0.4, \mathrm{SD}=0.7) . \mathrm{A}$ mixed ANOVA was conducted to illustrate differences in cortisol concentrations between the 
cortisol responders and non-responders. Expectedly, there was a main effect of group $\left(F_{(1}\right.$, $\left.\left.{ }_{65}\right)=63.127, \mathrm{p}<.001\right)$ and a significant interaction between time and group $\left(F_{(3.9,250.7)}=\right.$ 25.289, $p$ <.001). Pairwise comparisons indicated that cortisol concentrations were similar in the two groups at baseline and significantly different thereafter $(p<0.01)$. Cortisol concentrations in the responders and non-responders are shown in Figure 2.

\section{Insert Figure 2 about here}

Raw values for postural sway ranged from 0.77 to $9.46(\mathrm{~cm} / \mathrm{sec})$. A paired t-test showed significant differences in postural sway $(t=7.256$, df $=67, p<.001)$, with greater sway in the visual motion $(\mathrm{M}=3.36, \mathrm{SD}=1.89 \mathrm{~cm} / \mathrm{sec})$ compared to the static $(\mathrm{M}=1.40, \mathrm{SD}=0.39$ $\mathrm{cm} / \mathrm{sec}$ ) condition. Postural sway in the motion condition increased an average $134 \%$ compared to the static condition. Postural sway in the visual static $(t=0.247, d f=66, p=$ .806 ) or visual motion $(\mathrm{t}=1.542 \mathrm{df}=66, \mathrm{p}=.128$ ) conditions did not differ between the cortisol responders and non-responders. TSST-G group size or order participants completed the TSST was not related with cortisol reactivity or cortisol responder status $(p>.05)$. Nor were there any differences in the demographics or health variables measured in this study $(p$ $>0.05)$.

Regression analysis was conducted to examine the effect of postural sway on cortisol reactivity, with cortisol responder status modelled as a possible modulator. In the visual static condition, postural sway was not associated with cortisol reactivity $(F=.905$, df $=1$, $64, p=.345)$ nor did it interact with cortisol responder status $(F=.437$, df $=1,64, p=.511)$. In the visual motion condition, postural sway was negatively associated with cortisol reactivity $(F=4.436$, $d f=1,64, p=.039)$, with greater sway when viewing motion associated with lower cortisol reactivity. There was an interaction between postural sway and cortisol responder status $(F=5.163$, $d f=1,64, p=.026)$, suggesting that the association between postural sway and cortisol reactivity was observed in the cortisol responder group only (see Figure 3 for illustration). Menstrual cycle, age, smoking status, BMI did not interact with cortisol or postural sway, but to check that the relationship between cortisol reactivity and postural sway was not influenced by these variables they were entered as additional predictors. They did not predict cortisol reactivity and the main results (i.e. postural sway was negatively associated with cortisol reactivity) were unchanged.

\section{Insert Figure 3 about here}

\section{Discussion}


Visual dependence was examined in relation to stress-induced cortisol reactivity, an index of HPA axis function. The TSST-G was used to induce psychosocial stress in healthy young females. Despite no differences in basal cortisol concentrations a negative relationship between stress-induced cortisol reactivity and visual dependency indicated that those with the largest cortisol response showed less postural sway when exposed to visual motion. These findings were independent of menstrual phase, age, smoking status and BMI. The findings suggest that greater visual dependence in postural control is associated with attenuated cortisol secretion to a psychosocial stressor. This suggests that non-clinical differences in vestibular function, as indicated by greater visual dependence in healthy participants, are associated with dysregulation of the HPA axis activity, as indicated by lower cortisol reactivity to psychosocial stress.

Average sway did not differ between cortisol non-responders and responders; there were similar distributions between the groups. Moreover, in this sample neither age, menstrual phase, smoking status nor BMI predicted cortisol responding status. This is an interesting observation, suggestive of a possibly random nature of cortisol non-responding, and is consistent with a range of literature indicating no sociodemographic or physiological differences in cortisol responder and non-responder populations (Hamer and Steptoe, 2012; Seeman et al., 2001; Steptoe et al., 2017). Indeed, desynchrony between the affective and the salivary cortisol response to acute stress has been documented, with associations detected in only in about one quarter of the studies (Campbell and Ehlert, 2012). This desynchrony may be due to the underlying ultradian nature of cortisol secretion. Rodent studies have demonstrated marked phase-dependent effects in response to stress. If stress exposure coincides with the falling phase of the ultradian burst there are markedly attenuated $\mathrm{ACTH}$, corticosterone and behavioural responses (Lightman and ConwayCampbell, 2010). It was not possible to assess the ultradian phase of participants in this study so the timing of stress induction relative to the rising or falling ultradian bursts is unknown and will be random. This is likely to account for the failure to characterize nonresponders in the wider literature. The proportion of cortisol responders in this study (35.3\%) is similar to other studies in laboratory-based stress tasks that have examined responders and non-responders separately in their analyses. For example, Steptoe et al. (2017) and Hamer and Steptoe (2012) found $40.0 \%$ and $37.9 \%$ response rates using the lower $1 \mathrm{nmol} / \mathrm{l}$ threshold. It is important to note that the high percentage of non-responders in this sample might be because it was an all-female sample. Previous research suggests that males are more likely to show a cortisol response to the TSST (e.g. Eisenberger et al., 2007; Uhart et 
al., 2006). A 2.5nmol/l threshold has been widely applied in HPA axis related research (Wust et al., 2000). However, more recently to differentiate responders from non-responders, limiting inclusion of false positives, a threshold of $1.5 \mathrm{nmol} / \mathrm{l}$ has been proposed as more reflective of a cortisol secretory episode (Miller et al., 2013). In the current study, we used the higher threshold of $2.0 \mathrm{nmol} / \mathrm{l}$ cortisol response (Schwabe et al., 2008) which is commonly used within the literature (Bellingrath and Kudielka, 2008). It is important to emphasize that cortisol non-responding is distinct from low cortisol responding.

Blunted cortisol reactivity to stress is increasingly recognized as an index of dysregulated HPA axis function (Carroll et al., 2017). It is proposed that optimal responses to stress occur within a normal range for a given threat and that deviation from the norm signals poor systems integration. Just as exaggerated stress responses have negative consequences for health, blunted stress responses also signal poor homeostatic regulation, with a different set of consequences for health and behaviour (Carroll et al., 2017). Indeed, it has been suggested that larger cortisol stress reactivity might be protective against stress-related depression. Whilst acute stress reduces nucleus accumbens activation in response to reward cues (anhedonia), cortisol suppresses this relationship in that high cortisol responses are linked to stronger brain response to reward (Oei et al., 2014). Consistent with this suggestion, patients with clinical depressive disorder exhibit blunted cortisol reactivity to stress (Burke et al., 2005). The reduced cortisol response to stress in participants with greater visual dependency demonstrated here, parallels that observed for a range of adverse behavioural and health outcomes including cognitive impairment and anxiety (Domes et al., 2002), both of which have been associated with impaired vestibular function (Redfern et al., 2001).

The vestibular system has bi-directional links with the HPA axis and vestibular disorders are commonly associated with psychological symptoms such as anxiety. Stimulation of the vestibular pathways activates paraventricular neurons in the guinea pig and rat brain and retrograde viral tracing has demonstrated direct vestibulo-paraventricular and paraventricular-vestibular pathways in rodent brain (see Saman et al., 2012 for a review). Evidence of these pathways can be seen in humans where caloric stimulation of the vestibular system induces elevations in serum cortisol (Dagilas et al., 2005). Interestingly, larger cortisol responses to vestibular stimulation have been found in individuals with low susceptibility to motion sickness. Consequently, it is suggested that the cortisol response promotes appropriate vestibular compensation (see Saman et al., 2012 for a discussion), with the hippocampus playing a particularly important role (Eulenburg et al., 2010). It is 
noteworthy therefore that larger hippocampal volume has been associated with significantly greater cortisol reactivity in response to the TSST (Pruessner et al., 2007). This finding is consistent with the result presented here in as much as larger cortisol responses to the TSST-G was associated with less increases in postural sway during exposure to visual motion.

It is important to consider the limitations of this study. Firstly, in order to remove sex, age and pathology as potential confounds, all participants were healthy young females. Whether these findings are representative of effects in males, other age groups and conditions of vestibular dysfunction or chronic stress cannot be deduced. Higher basal cortisol levels has been shown to predict better tolerance to motion sickness induced in the laboratory (Meissner et al., 2009), however, we selected to induce HPA axis activation using the TSST$\mathrm{G}$ as this protocol most represents stress experienced in daily lives (i.e. social-evaluative threat and uncontrollability). Indeed, other laboratory protocols that may be capable of inducing a cortisol response, such as, laboratory induced motion sickness, would also enable examination of postural control in relation to HPA axis function. It may be worthwhile to examine associations between HPA axis and vestibular function in participants with vestibular disorder, older age, anxiety disorder, depression and mild cognitive impairment. In addition, future investigations could examine whether individuals with motion sensitivities, such as motion sickness, which tend to be more visually dependent, also demonstrate blunted cortisol reactivity to stress.

\section{Conclusions}

In conclusion, we have demonstrated that reduced cortisol reactivity to psychosocial stress in healthy females is related to greater visual dependence in postural control. In this study of healthy participants, with no reported vestibular disorder or psychological comorbidity, we show that greater visual dependency in postural control was related to attenuated HPA axis function. These findings add to the growing body of evidence linking reduced cortisol reactivity to poor homeostatic regulation with potential long-term negative health and behavioural outcomes.

\section{References}


Almela, M., Hidalgo, V., van der Meij, L., Pulopulos, M.M., Villada, C., Salvador, A., 2014. A low cortisol response to acute stress is related to worse basal memory performance in older people. Front. Aging Neurosci. 6, 157.

Bellingrath, S., Kudielka, B.M., 2008. Effort-reward-imbalance and overcommitment are associated with hypothalamus-pituitary-adrenal (HPA) axis responses to acute psychosocial stress in healthy working schoolteachers. Psychoneuroendocrinology 33, 1335-1343.

Bigelow, R.T., Agrawal, Y., 2015. Vestibular involvement in cognition: Visuospatial ability, attention, executive function, and memory. J. Vestib. Res.-Equilib. Orientat. 25, 73-89.

Bradley, A.J., Dinan, T.G., 2010. A systematic review of hypothalamic-pituitary-adrenal axis function in schizophrenia: implications for mortality. J. Psychopharmacol. 24, 91-118.

Burke, H.M., Davis, M.C., Otte, C., Mohr, D.C., 2005. Depression and cortisol responses to psychological stress: A meta-analysis. Psychoneuroendocrinology 30, 846-856.

Campbell, J., Ehlert, U., 2012. Acute psychosocial stress: Does the emotional stress response correspond with physiological responses? Psychoneuroendocrinology 37,1111 1134.

Carroll, D., Ginty, A.T., Whittaker, A.C., Lovallo, W.R., de Rooij, S.R., 2017. The behavioural, cognitive, and neural corollaries of blunted cardiovascular and cortisol reactions to acute psychological stress. Neurosci. Biobehav. Rev. 77, 74-86.

Chan, O., Inouye, K., Riddell, M., Vranic, M., Matthews, S., 2003. Diabetes and the hypothalamo-pituitary-adrenal (HPA) axis. Minerva endocrinologica 28, 87-102. Cousins, S., Cutfield, N.J., Kaski, D., Palla, A., Seemungal, B.M., Golding, J.F., Staab, J.P., Bronstein, A.M., 2014. Visual Dependency and Dizziness after Vestibular Neuritis. PLoS One $9,6$.

Cousins, S., Kaski, D., Cutfield, N., Arshad, Q., Ahmad, H., Gresty, M.A., Seemungal, B.M., Golding, J., Bronstein, A.M., 2017. Predictors of clinical recovery from vestibular neuritis: a prospective study. Ann. Clin. Transl. Neurol. 4, 340-346.

Dagilas, A., Kimiskidis, T., Aggelopoulou, M., Kapaki, T., Fitili, C., Libitaki, G., Papagiannopoulos, T., Kazis, D., Kazis, A., Aidonis, A., 2005. Changes in blood neurotransmitter and steroid levels during evoked vertigo. Otol. Neurotol. 26, 476-480. Dickerson, S.S., Kemeny, M.E., 2004. Acute stressors and cortisol responses: A theoretical integration and synthesis of laboratory research. Psychol. Bull. 130, 355-391.

Domes, G., Heinrichs, A., Reichwald, U., Hautzinger, M., 2002. Hypothalamic-pituitaryadrenal axis reactivity to psychological stress and memory in middle-aged women: high responders exhibit enhanced declarative memory performance. Psychoneuroendocrinology 27, 843-853.

Eisenberger, N.I., Taylor, S.E., Gable, S.L., Hilmert, C.J., Lieberman, M.D., 2007. Neural pathways link social support to attenuated neuroendocrine stress responses. Neuroimage 35, 1601-1612.

Eulenburg, P.Z., Stoeter, P., Dieterich, M., 2010. Voxel-Based Morphometry Depicts Central Compensation after Vestibular Neuritis. Ann. Neurol. 68, 241-249.

Faravelli, C., Lo Sauro, C., Lelli, L., Pietrini, F., Lazzeretti, L., Godini, L., Benni, L., Fioravanti, G., Alina Talamba, G., Castellini, G., 2012. The role of life events and HPA axis in anxiety disorders: a review. Current pharmaceutical design 18, 5663-5674.

Furman, J.M., Jacob, R.G., 2001. A clinical taxonomy of dizziness and anxiety in the otoneurological setting. J. Anxiety Disord. 15, 9-26.

Goodman, W., Janson, J., Wolf, J., 2017. Meta-analytical assessment of the effects of protocol variations on cortisol responses to the Trier Social Stress Test.

Psychoneuroendocrinology 80, 26-35.

Hamer, M., Steptoe, A., 2012. Cortisol Responses to Mental Stress and Incident Hypertension in Healthy Men and Women. J. Clin. Endocrinol. Metab. 97, E29-E34. Hartmann, A., Veldhuis, J.D., Deuschle, M., Standhardt, H., Heuser, I., 1997. Twenty-four hour cortisol release profiles in patients with Alzheimer's and Parkinson's disease compared 
to normal controls: ultradian secretory pulsatility and diurnal variation. Neurobiology of aging 18, 285-289.

Jeon, H.J., Cho, M.J., Cho, S.J., Kim, S.U., Park, S.K., Jun, S.K., Jeon, J.Y., Hahm, B.J., 2007. Quantitative analysis of ataxic gait in patients with schizophrenia: The influence of age and visual control. Psychiatry Res. 152, 155-164.

Kirschbaum, C., Pirke, K.M., Hellhammer, D.H., 1993. TheTrier Social Stress Test - A tool for investigating psychobiological stress responses in a laboratory setting.

Neuropsychobiology 28, 76-81.

Kudielka, B.M., Hellhammer, D.H., Wust, S., 2009. Why do we respond so differently? Reviewing determinants of human salivary cortisol responses to challenge.

Psychoneuroendocrinology 34, 2-18.

Lightman, S.L., Conway-Campbell, B.L., 2010. The crucial role of pulsatile activity of the HPA axis for continuous dynamic equilibration. Nat. Rev. Neurosci. 11, 710-718.

Markia, B., Kovacs, Z.I., Palkovits, M., 2008. Projections from the vestibular nuclei to the hypothalamic paraventricular nucleus: morphological evidence for the existence of a vestibular stress pathway in the rat brain. Brain Struct. Funct. 213, 239-245.

Martin, T., Moussay, S., Bulla, I., Bulla, J., Toupet, M., Etard, O., Denise, P., Davenne, D., Coquerel, A., Quarck, G., 2016. Exploration of Circadian Rhythms in Patients with Bilateral Vestibular Loss. PLoS One 11, 20.

Meissner, K., Enck, P., Muth, E., Kellermann, S., Klosterhalfen, S., 2009. Cortisol levels predict motion sickness tolerance in women but not in men. Physiology \& behavior 97, 102106.

Miller, R., Plessow, F., Kirschbaum, C., Stalder, T., 2013. Classification criteria for distinguishing cortisol responders from nonresponders to psychosocial stress: evaluation of salivary cortisol pulse detection in panel designs. Psychosomatic Medicine 75, 832-840. Miller, R., Wojtyniak, J.-G., Weckesser, L.J., Alexander, N.C., Engert, V., Lehr, T., 2018. How to disentangle psychobiological stress reactivity and recovery: A comparison of modelbased and non-compartmental analyses of cortisol concentrations.

Psychoneuroendocrinology 90, 194-210.

Oei, N.Y.L., Both, S., van Heemst, D., van der Grond, J., 2014. Acute stress-induced cortisol elevations mediate reward system activity during subconscious processing of sexual stimuli. Psychoneuroendocrinology 39, 111-120.

Pierce, M., Pritchard, L., 2016. Lower stress-reactive cortisol in female veterans associated with military status but not PTSD. Stress 19, 486-491.

Pruessner, M., Pruessner, J.C., Hellhammer, D.H., Pike, G.B., Lupien, S.J., 2007. The associations among hippocampal volume, cortisol reactivity, and memory performance in healthy young men. Psychiatry Res. Neuroimaging 155, 1-10.

Raffington, L., Prindle, J., Keresztes, A., Binder, J., Heim, C., Shing, Y.L., 2018. Blunted cortisol stress reactivity in low-income children relates to lower memory function.

Psychoneuroendocrinology 90, 110-121.

Razzak, R.A., Hussein, W., 2016. Postural visual dependence in asymptomatic type 2 diabetic patients without peripheral neuropathy during a postural challenging task. J.

Diabetes Complications 30, 501-506.

Redfern, M.S., Yardley, L., Bronstein, A.M., 2001. Visual influences on balance. J. Anxiety Disord. 15, 81-94.

Saman, Y., Bamiou, D.E., Gleeson, M., Dutia, M.B., 2012. Interactions between Stress and Vestibular Compensation - A Review. Frontiers in Neurology 3, 116.

Schwabe, L., Haddad, L., Schachinger, H., 2008. HPA axis activation by a socially evaluated cold-pressor test. Psychoneuroendocrinology 33, 890-895.

Seeman, T.E., Singer, B., Wikinson, C.W., McEwen, B., 2001. Gender differences in agerelated changes in HPA axis reactivity. Psychoneuroendocrinology 26, 225-240.

Skoluda, N., Strahler, J., Schlotz, W., Niederberger, L., Marques, S., Fischer, S., Thoma, M., Spoerri, C., Ehlert, U., Nater, U., 2015. Intra-individual psychological and physiological 
responses to acute laboratory stressors of different intensity. Psychoneuroendocrinology 51, 227-236.

Smyth, N., Hucklebridge, F., Thorn, L., Evans, P., Clow, A., 2013. Salivary cortisol as a biomarker in social science research. Social and Personality Psychology Compass 7, 605625.

Smyth, N., Thorn, L., Oskis, A., Hucklebridge, F., Evans, P., Clow, A., 2015. Anxious attachment style predicts an enhanced cortisol response to group psychosocial stress. Stress 18, 143-148.

Steptoe, A., Hamer, M., Lin, J., Blackburn, E.H., Erusalimsky, J.D., 2017. The Longitudinal Relationship Between Cortisol Responses to Mental Stress and Leukocyte Telomere Attrition. J. Clin. Endocrinol. Metab. 102, 962-969.

Uhart, M., Chong, R.Y., Oswald, L., Lin, P.-I., Wand, G.S., 2006. Gender differences in hypothalamic-pituitary-adrenal (HPA) axis reactivity. Psychoneuroendocrinology 31, 642652.

von Dawans, B., Kirschbaum, C., Heinrichs, M., 2011. The Trier Social Stress Test for Groups (TSST-G): A new research tool for controlled simultaneous social stress exposure in a group format. Psychoneuroendocrinology 36, 514-522.

Wust, S., Wolf, J., Hellhammer, D., Federenko, I., Schommer, N., Kirschbaum, C., 2000. The cortisol awakening response - normal values and confounds. Noise and Health 2, 79-88.

Yamanaka, T., Sasa, M., Amano, T., Miyahara, H., Matsunaga, T., 1995. Role of Glucocorticoid in vestibular compensation in relation to activation of vestibular nucleus neurons. Acta Oto-Laryngol., 168-172.

Zänkert, S., Bellingrath, S., Wüst, S., Kudielka, B.M., 2018. HPA axis responses to psychological challenge linking stress and disease: What do we know on sources of intraand interindividual variability? Psychoneuroendocrinology.

Table 1. Descriptive statistics for demographic and health characteristics $(n=68)$

\begin{tabular}{lll}
\hline Variables & Descriptives & Min-Max \\
& & \\
\hline Age in years M (SD) & $20.04(2.52)$ & $18-31$ \\
BMI M (SD) & $22.53(5.70)$ & $15.80-53.40$ \\
Ethnicity N (\%) & \\
Asian & $33(49.3)$ & \\
European White & $23(33.8)$ & \\
African Caribbean & $4(5.9)$ & \\
Mixed race & $7(10.3)$ & \\
Smoking status \% non- & $50(73.5)$ & \\
smoker & & \\
Menstrual cycle phase \% & $20(29.4)$ \\
luteal & \\
Oral Contraceptives \% taking & $2(2.9)$ \\
\hline
\end{tabular}




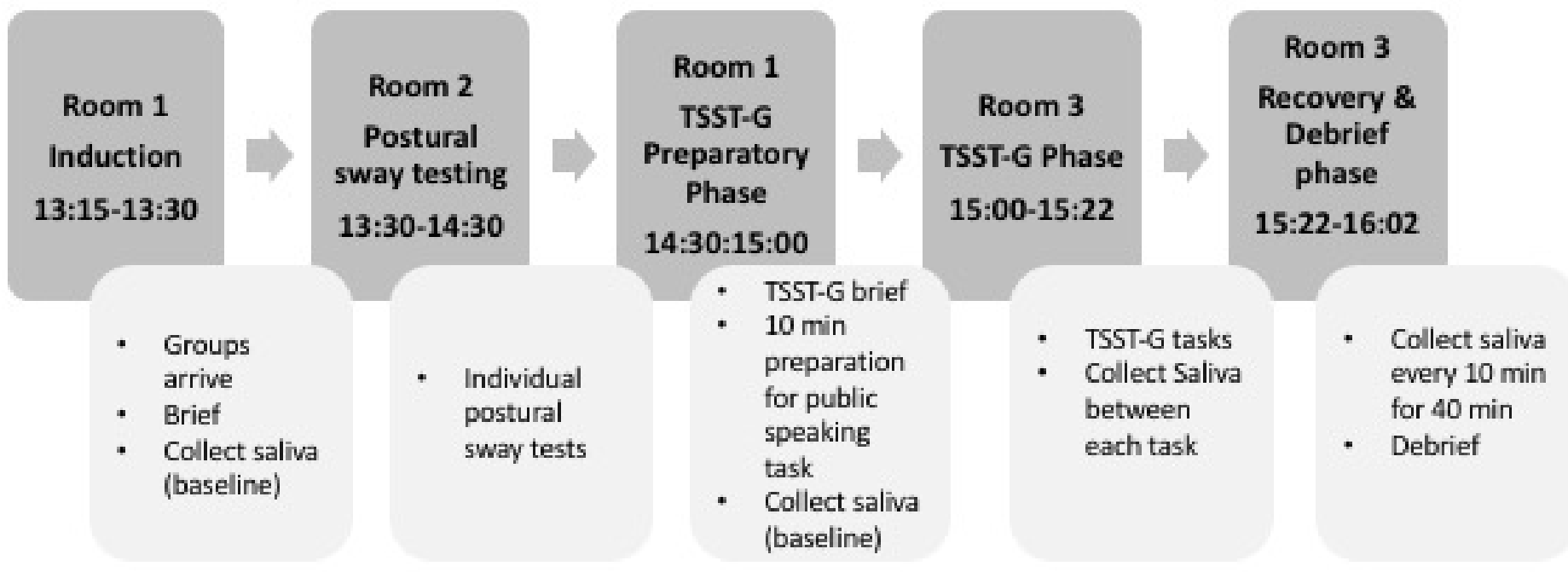

Figure 1. Experimental timeline based on groups of up to 6 participants. 


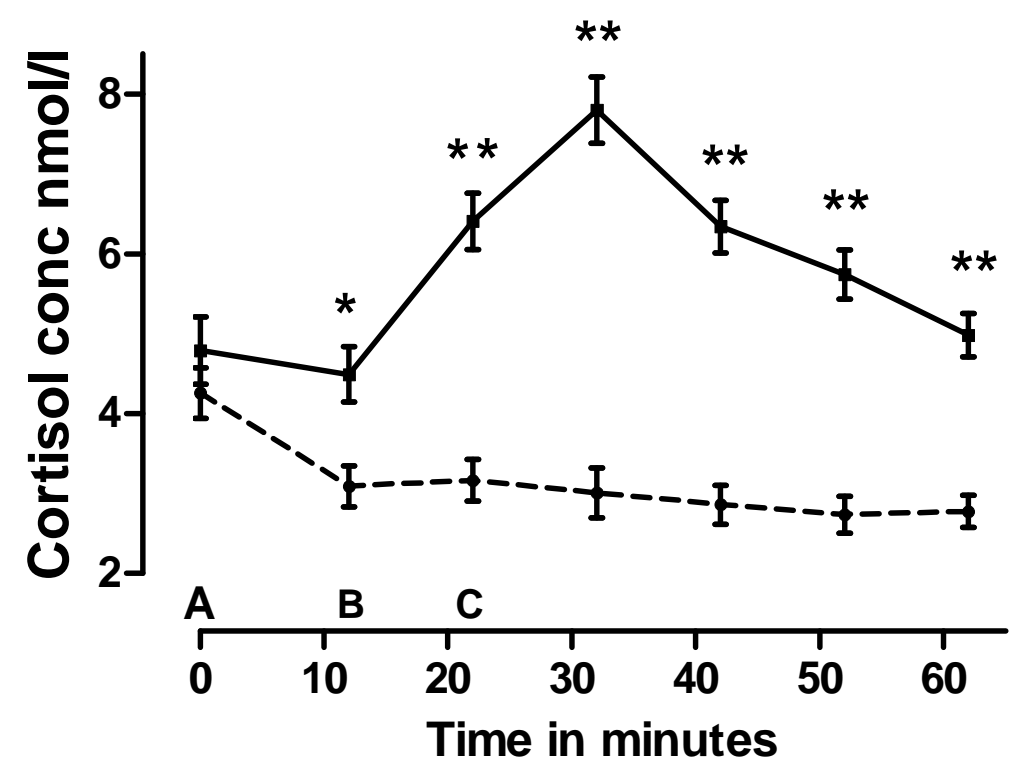

Figure 2. Mean \pm SEM salivary cortisol concentrations in cortisol responders (solid line) and non-responders (dashed line). Responders defined as showing more than $2.0 \mathrm{nmol} / \mathrm{l}$ increase from their individual base to maximum concentration. A: average baseline, before onset of The TSST-G; B mid-way through the TSST-G; C immediately after the end of the TSST-G. * $p<0.01 ;{ }^{* *} p<0.001$ 


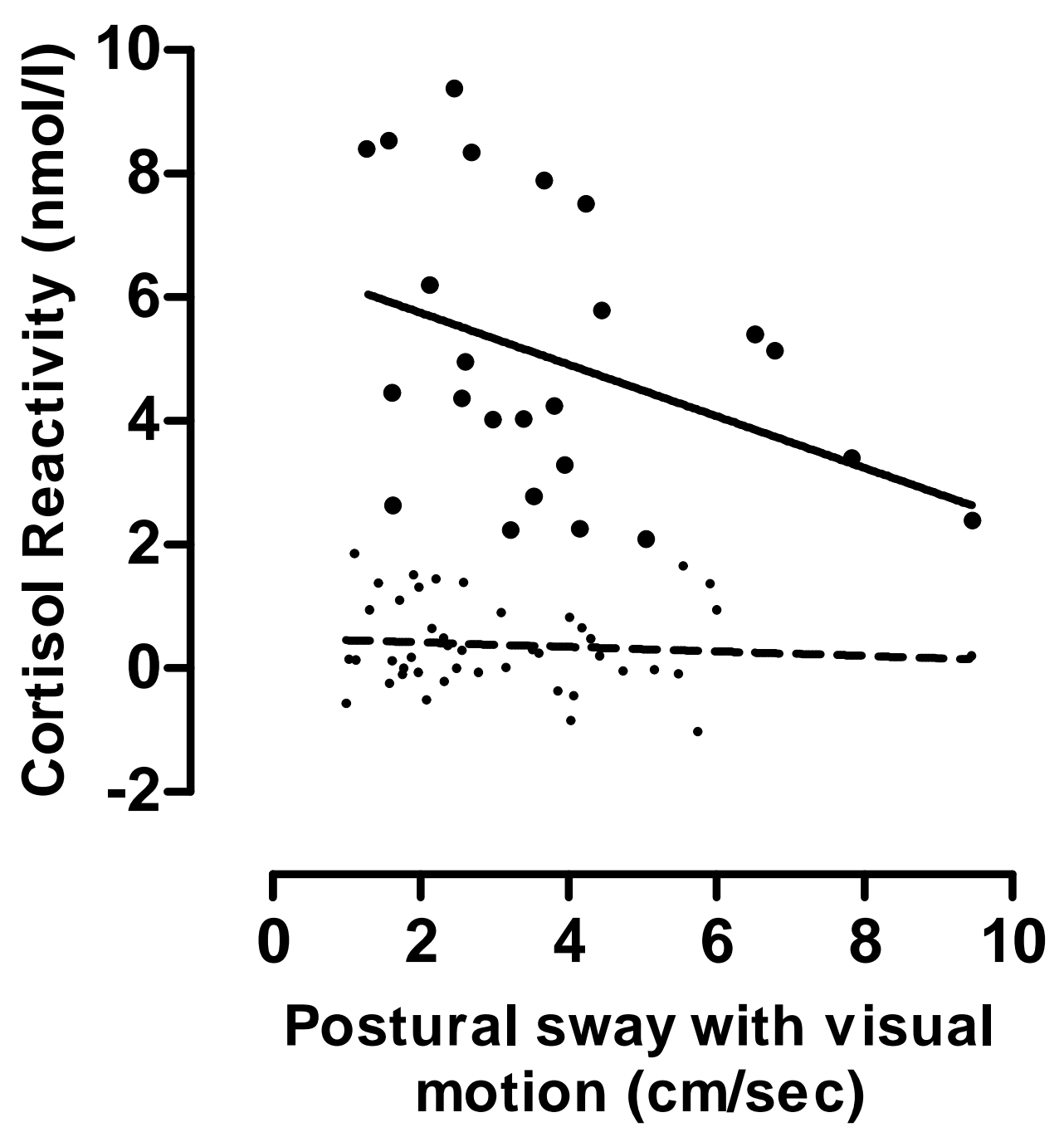

Figure 3. Interaction between cortisol responders (solid line) and non-responders (dashed line) and increased sway path length (cm/sec) with visual motion (i.e. visual dependence). Responders defined as showing more than $2.0 \mathrm{nmol} / \mathrm{l}$ increase from their individual base to maximum concentration. 